\title{
Predictors of poor sleep quality among Lebanese university students: association between evening typology, lifestyle behaviors, and sleep habits
}

This article was published in the following Dove Press journal:

Nature and Science of Sleep

13 January 2014

Number of times this article has been viewed

\author{
Colette S Kabrita' \\ Theresa A Hajjar-Muça² \\ Jeanne F Duffy ${ }^{3}$ \\ 'Department of Sciences, \\ ${ }^{2}$ Department of Mathematics and \\ Statistics, Faculty of Natural and \\ Applied Sciences, Notre Dame \\ University - Louaize, Zouk Mosbeh, \\ Lebanon; ${ }^{3}$ Division of Sleep Medicine, \\ Department of Medicine, Brigham and \\ Women's Hospital, Harvard Medical \\ School, Boston, MA, USA
}

\begin{abstract}
Adequate, good night sleep is fundamental to well-being and is known to be influenced by myriad biological and environmental factors. Given the unavailability of sleep data about Lebanon, the cultural shifts and socioeconomic pressures that have affected many aspects of society, particularly for students and working adults, as well as our understanding of sleep in university students in other countries, we conducted a national study to assess sleep quality and factors contributing to sleep and general health in a culture-specific context. A self-filled questionnaire, inquiring about sociodemographics, health-risk behaviors, personal health, and evaluating sleep quality and chronotype using standard scales was completed by 540 students at private and public universities in Lebanon. Overall, they reported sleeping 7.95 \pm 1.34 hours per night, although $12.3 \%$ reported sleeping $<6.5$ hours and more than half scored in the poor-sleeper category on the Pittsburgh Sleep Quality Index (PSQI). Sleep timing differed markedly between weekdays and weekends, with bedtimes and wake-up times delayed by 1.51 and 2.43 hours, respectively, on weekends. While most scored in the "neither type" category on the MorningnessEveningness Questionnaire (MEQ), 24.5\% were evening types and 7.3\% were morning types. MEQ score was significantly correlated with smoking behavior and daily study onset, as well as with PSQI score, with eveningness associated with greater number of cigarettes, later study times, and poor sleep. We conclude that the prevalence of poor sleep quality among Lebanese university students is associated with reduced sleep duration and shifts in sleep timing between weekdays and weekends, especially among evening types. While chronotype and certain behavioral choices interact to affect sleep dimensions and quality, raising awareness about the importance of obtaining adequate nighttime sleep on daily performance and avoiding risky behaviors may help Lebanese students make better choices in school and work schedules.
\end{abstract}

Keywords: bedtime irregularities, chronotype, Lebanon, PSQI, sleep duration, behavioral habits

\section{Introduction}

Sleep is part of a daily biological rhythm that is indispensable for promoting health and optimal bodily function. ${ }^{1}$ In humans, it is consolidated to the nocturnal phase of the 24-hour time scale due to an intricate interaction of two systems: a circadian timing system and a sleep homeostat. ${ }^{2}$ The former promotes wakefulness during the daytime and sleep at night. In contrast, the sleep-homeostatic component is dependent on the sleep-wake history of the person, with a drive for sleep (or sleep pressure) that builds up during wakefulness. ${ }^{3}$

Despite its biological necessity, sleep has been traded off in modern societies to accommodate social and work schedules. Many studies have shown that
Correspondence: Colette S Kabrita Department of Sciences, Faculty of Natural and Applied Sciences, Notre Dame University - Louaize, PO Box 72, Zouk Mikael,

Zouk Mosbeh, Lebanon

Tel +96| 9208728

Fax +9619218 77I

Email ckabrita@ndu.edu.lb 
sleep disturbance and poor sleep quality have adverse physiological ${ }^{4,5}$ consequences on increasing human morbidity and mortality. ${ }^{6}$ The mechanisms underlying sleep disturbance, commonly noted among adolescents and emerging adults, are not very well understood; however, a number of biobehavioral and environmental factors interact to influence sleep patterns. ${ }^{7}$ Developmental changes that occur at puberty, due to an alteration in the circadian timing system, partly mediate certain changes in adolescent sleep, such as delayed bedtimes throughout the week, leading to short sleep duration on schooldays, when rise time is determined by school start time, and extended sleep on weekends, when there are fewer morning social requirements. ${ }^{8,9}$ Recovery sleep on weekends is a homeostatic compensatory mechanism for the sleep debt created on weekdays, while phase delays in bedtimes are hypothesized to result from changes in the circadian timing system. ${ }^{10}$ Circadian typology (chronotype) is another influence on sleep habits, because individuals show variability in their sleep-wake timing preferences. ${ }^{11}$ These differences are partly innate, but are also shaped by environmental factors, such as daily light exposure (both natural and artificial), and may also be influenced by the seasonal photoperiod and the longitude and latitude of residence. ${ }^{12,13}$ Late chronotypes are at highest risk of sleep curtailment and disturbance, due to misalignment between their social and biological time. ${ }^{14}$ Additional factors that may interact with chronotype to affect sleep include such lifestyle habits as work schedules ${ }^{15}$ and socioeconomic stresses. ${ }^{16,17}$

University students are vulnerable to sleep disturbance ${ }^{18}$ due to multiple environmental factors that affect their sleep-wake behavior. These include reduced or eliminated parental influence and the freedom to self-select bedtime, increased academic demands, economic stresses, and the increased number of hours spent working and/or indulged in extracurricular activities. ${ }^{7}$ Such behaviors are associated with alteration in one or more of the sleep dimensions, such as phase delays in sleep-wake timing and reduction in total sleep time, as well as deterioration of sleep quality and daytime functioning. ${ }^{19-22}$ Insufficient and/or nonrestorative sleep has been shown to impact neurocognitive, psychological, ${ }^{23}$ and academic well-being. ${ }^{24-26}$ Kelly et $\mathrm{al}^{27}$ investigated the relationship between sleep quality and reported grade-point average (GPA) of undergraduate university students, and found that short-sleepers (total sleep time $\leq 6$ hours/night) had significantly lower GPAs than long-sleepers (total sleep time $\geq 9$ hours/night). The poor academic performance associated with short sleep durations was linked to reduced concentration ability on educational material, lower self-efficacy, and even possible psychological difficulties.

While there has been extensive investigation of the biological, psychological, and academic significance of adequate sleep, very little of that work has been done in the Arab world. Two studies addressing sleep patterns and problems of university students in the Middle East have been published. Sweileh et $\mathrm{al}^{28}$ showed that Palestinian undergraduate students reported an average of 6.4 hours of nighttime sleep, more than half went to bed before midnight, and $9.8 \%$ were poor sleepers. In that study, daytime naps were reported among $74.5 \%$ of respondents. Another study considered the sleeping habits of Saudi Arabian Muslim medical students during the fasting month of Ramadan. ${ }^{29}$ That study showed that students had delayed sleep-wake schedules, reduced total sleep time, and about two-thirds had daytime naps during Ramadan. Variations in self-reported sleep problems, however, exist among adults of different ethnic and cultural backgrounds. ${ }^{30,31}$ While a relatively small country (a population of approximately 4,140,289, according to the World Bank 2013 estimate), Lebanon has great cultural and demographic diversity compared to other Middle Eastern countries. Furthermore, it has undergone many cultural shifts (including changes in eating habits, ${ }^{32}$ increased use of narghile [also termed hookah or "hubble bubble", a water pipe used for smoking flavored tobacco], and widespread use of electronic devices) and socioeconomic changes in the postwar period that may have implications for sleep, as shown in other studies. ${ }^{33}$ The siesta culture is also uncommon in modern Lebanese society. Therefore, given the absence of sleep information about Lebanon and to understand the sleep habits of young Lebanese adults and whether they, like young adults in Western countries, experience insufficient sleep quantity and poor sleep quality, we conducted the present study.

The aims of the present study were to assess sleep quality among university students in Lebanon in a culture-specific context, and to investigate how circadian typology may interact with sleep habits and behavioral lifestyle choices to affect sleep quality. Our larger goal is to use our understanding of the sleep profile of students to raise awareness of the health and academic importance of sleep in order to improve the overall well-being of university students in Lebanon.

\section{Materials and methods \\ Participants and procedures}

This study was conducted at six private and public universities in different regions in Lebanon in order to secure cultural diversity, both regional and religious. To ensure student 
variability with regards to age, sex, major, and level of education, up to eight sections of an undergraduate English general requirement course (as applicable to the university), hosting an average of 25 students each, were randomly selected from each university for data collection.

Prior to data collection, questionnaires were sent to each of the selected universities, to be reviewed and approved by the local ethics board for research on human subjects (or other concerned authorities) to assure compliance with international guidelines.

For any given university, sampling was done either during the mid-fall or early spring semester (with the entire data collection extending from spring 2010 through spring 2011). With the help of two trained field researchers and the respective course instructors, full-time students were orally briefed on the purpose and importance of the study and were encouraged to complete the questionnaires (optional). Only questionnaires from respondents in the age range of 17-25 years were included in the final analysis. Participation in the study was voluntary, and did not involve financial or other compensation or course credit.

\section{Study instruments}

A pretested questionnaire (original English version) consisting of four parts was used. The first part covered demographic (age, sex, etc), academic (major, education level, study habits, etc), and occupational and socioeconomic (employment status, total hours of work per week, perceived socioeconomic level, etc) questions. The second part focused on health behaviors, and included questions about smoking, alcohol consumption, diet (including caffeine use), exercise, and sleep.

The third part emphasized personal health, and inquired about body weight, general health status, medical condition, sleep disorders, medication and supplement use, in addition to psychoactive drug use. The fourth part included a number of standardized tests, such as the Pittsburgh Sleep Quality Index (PSQI) ${ }^{34}$ to assess sleep quality and calculate sleep parameters (sleep duration), and the Morningness-Eveningness Questionnaire (MEQ). ${ }^{35}$

\section{Statistical analysis and questionnaire scoring}

Data were entered, edited, and analyzed using the SPSS version 11.5.1 for Windows (SPSS Inc., Chicago, IL, USA). Descriptive statistics were used to describe the demographic characteristics of the sample and to present the prevalence of behavioral habits, health conditions, sleep disorders, and chronotype.
Analysis of variance (ANOVA, for chronotype group comparisons of sleep duration and sleep-wake timing) along with effect size $\eta^{2} \rho$, Student's $t$-test (for comparison of sleep duration and sleep-wake habits between weekdays and weekends), and bivariate correlation tests (to study correlations between PSQI, MEQ, and other behavioral and sleep habits) were used.

Standard scoring was used on the MEQ, and subjects were categorized into morning, neither, or evening types. Subjects who scored as moderately morning or evening were included in the morning and evening groups, respectively. A PSQI global score above 5 was used to categorize poor sleep, and sleep duration $<6.5$ hours was categorized as insufficient sleep. A sleep time difference of more than 1 hour between weekdays and weekends was considered a sign of "social jet lag", ${ }^{14}$ and was used to categorize irregular bedtimes.

\section{Results \\ Respondents' characteristics Demographic and socioeconomic profile}

A total of 540 students completed the questionnaires. Their average age was $19.85 \pm 1.51$ years. Approximately half were females (50.6\%), sophomores (53.4\%), and the majority was Lebanese and/or of Lebanese descent (95.7\%).

Approximately a third of respondents (31.6\%) reported having a job, $74.3 \%$ of whom worked part-time. Out of the 106 who responded to the number of hours of part-time employment per week, $41.5 \%$ worked 12-30 hours per week, and $29.2 \%$ worked more than 30 hours per week. Eleven percent of respondents reported low socioeconomic status. Table 1 summarizes the demographic and socioeconomic characteristics of respondents.

\section{Personal lifestyle habits}

Less than half the respondents (42.9\%) reported smoking cigarettes and/or narghile, around half (49.3\%) reported drinking alcohol (4.9\% of whom drank on a daily basis), $88.1 \%$ consumed caffeinated beverages, and $56.6 \%$ exercised. For $40 \%$ of respondents, the daily study onset was between $6 \mathrm{pm}$ and 6 am. Results are summarized in Table 2.

\section{Sleep behavior}

A total of $89.9 \%$ of respondents went to bed after $11 \mathrm{pm}$ on weekdays compared to $97.5 \%$ of respondents who did so on weekends $\left(P<0.001, \chi^{2}=25.630\right)$. Half of the respondents reported a difference in sleep timing of more than an hour between weekdays and weekends. The average nocturnal sleep duration (considering both weekdays and weekends) 
Table I Demographic and socioeconomic status of respondents

\begin{tabular}{llll}
\hline Variable & $\mathbf{n}$ & $\% \$$ & Mean \pm SD \\
\hline Age $(\mathrm{n}=539)$, years & & & \\
$\quad$ Males & 266 & 49.4 & $20.08 \pm 1.6 \mathrm{I}$ \\
$\quad$ Females & 273 & 50.6 & $19.68 \pm 1.39$ \\
Educational level $(\mathrm{n}=46 \mathrm{I})$ & & & \\
$\quad$ Sophomore & 246 & 53.4 & \\
$\quad$ Junior & 114 & 24.7 & \\
$\quad$ Senior & $10 \mathrm{I}$ & 21.9 & \\
Employment status $(\mathrm{n}=538)$ & & & \\
$\quad$ Employed & 170 & 31.6 & \\
Looking for a job & 64 & 11.9 & \\
$\quad$ Not employed & 304 & 56.5 & \\
Socioeconomic status $(\mathrm{n}=509)$ & & & \\
$\quad$ Low & 56 & 11.0 \\
$\quad$ Medium & 402 & 79.0 \\
$\quad$ High & $5 \mathrm{I}$ & 10.0 & \\
\hline
\end{tabular}

Note: $\$$ Valid percentages are reported.

Abbreviation: SD, standard deviation.

was $7.95 \pm 1.34$ hours, and $12.3 \%$ of respondents were categorized as having insufficient sleep. These results are presented in Table 2.

Of the 437 participants who fully responded to the MEQ, $68.2 \%$ were categorized as neither evening nor morning types, $24.5 \%$ as evening types, $7.3 \%$ as morning types.

Table 2 Personal lifestyle habits and sleep behavior of respondents

\begin{tabular}{|c|c|c|c|}
\hline Variable & $\mathbf{n}$ & $\% §$ & Mean \pm SD \\
\hline \multicolumn{4}{|c|}{ Smoking cigarettes/narghile $(n=539)$} \\
\hline Yes & 231 & 42.9 & \\
\hline No & 308 & 57.1 & \\
\hline \multicolumn{3}{|c|}{ Smoking cigarettes $(n=539)$} & Cigarettes/day: $12.74 \pm 10.30$ \\
\hline Yes & III & 20.6 & \\
\hline No & 428 & 79.4 & \\
\hline \multicolumn{3}{|c|}{ Smoking narghile $(\mathrm{n}=533)$} & Narghile/month: $9.79 \pm 10.33$ \\
\hline Yes & 169 & 31.7 & \\
\hline No & 364 & 68.3 & \\
\hline \multicolumn{4}{|c|}{ Study onset $(n=497)$} \\
\hline$>12$ am-6 am & 15 & 3.0 & \\
\hline$>6 \mathrm{am}-12 \mathrm{pm}$ & 130 & 26.2 & \\
\hline$>12 \mathrm{pm}-6 \mathrm{pm}$ & 168 & 33.8 & \\
\hline$>6 \mathrm{pm}-12 \mathrm{am}$ & 184 & 37.0 & \\
\hline \multicolumn{4}{|l|}{ Bedtime } \\
\hline Weekdays $(\mathrm{n}=5$ & & & $12: 10 \mathrm{am} \pm 83 \mathrm{~min}$ \\
\hline Weekends $(\mathrm{n}=$ & & & $1: 39 \mathrm{am} \pm 100 \mathrm{~min}$ \\
\hline \multicolumn{4}{|c|}{ Irregular bedtime between weekdays and weekends $(n=5 \mid 6)$} \\
\hline Yes & 259 & 50.2 & \\
\hline No & 257 & 49.8 & \\
\hline \multicolumn{4}{|c|}{ Nocturnal sleep duration, hours (SD) $(n=496)$} \\
\hline$<6.5$ & 61 & 12.3 & \\
\hline $6.5-8$ & 197 & 39.7 & \\
\hline$>8$ & 238 & 48.0 & \\
\hline
\end{tabular}

Note: ${ }^{\S}$ Valid percentages are reported.

Abbreviations: SD, standard deviation; min, minutes.
The PSQI global scores revealed that out of the 218 respondents, the majority $(58.7 \%)$ reported poor sleep quality $(>5)$, with an average PSQI score of $6.57 \pm 3.49$.

\section{Association between sleep and demographic characteristics and personal habits}

There was no significant relationship between age, sex, employment and socio-economic status, and PSQI. Behaviorally, exercise and caffeine consumption were not significantly related to PSQI either. Day length (duration of photoperiod) was not significantly correlated with sleep duration, sleep-wake timing on weekdays and weekends, or PSQI.

There was a significant negative correlation between PSQI and MEQ scores $(r=-0.183, P=0.011)$, indicating a greater incidence of poor sleep with greater eveningness. Sleep duration correlated negatively and significantly with PSQI global score $(r=-0.242, P<0.001)$, implying an association between reduced sleep length and poor sleep quality.

Sleep duration was extended by an average of 1 hour on weekends compared with weekdays $(8.66 \pm 1.76$ versus [vs] $7.71 \pm 1.46$ hours, respectively). This tendency was more notable among the evening-type group, and there was a significant difference in sleep duration among the chronotype groups on weekends $\left(F_{[2,417]}=4.957, P=0.007\right)$, but not weekdays (Figure 1).

Students had an average 1.51-hour delay in their bedtime (1:39 am \pm 100 minutes vs 12:08 am \pm 81 minutes $)$ and a 2.43-hour delay in their wake-up time between weekends and weekdays. The evening types had the greatest weekend bedtime delay of the three groups. One-way ANOVA revealed significant differences in sleep-wake habits among evening, neither, and morning groups on weekdays and weekends (Table 3).

Using the Student's $t$-test to compare sleep-wake timing between weekdays and weekends for each chronotype, results revealed a significant difference in bedtimes among the evening group ( $t=-9.708, P<0.001)$, neither $(t=-12.041$, $P<0.001)$, and morning types $(t=-2.925, P=0.005)$. Similarly, significant differences were observed for wake-up time between weekdays and weekends for each chronotype (evening, $t=-14.244, P<0.001$; neither, $t=-18.307, P<0.001$; morning, $t=-3.828, P<0.001)$.

\section{Study habits}

Study onset was negatively and significantly correlated with MEQ ( $r=-0.162, P<0.001)$, indicating that later study-time preferences are associated with eveningness. 


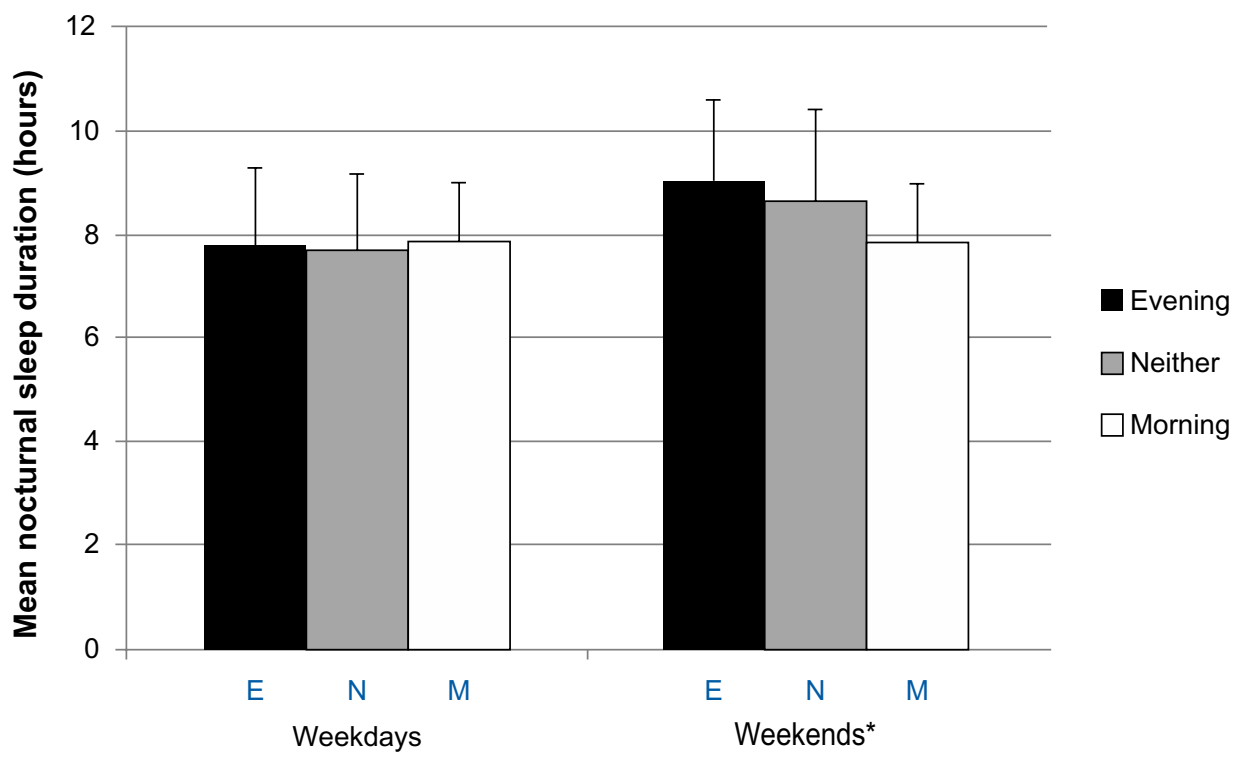

Figure I Mean nocturnal sleep duration (hours) on weekdays (left) and weekends (right) by chronotype. The weekend-weekday difference in sleep duration was significant in the $\mathrm{E}$ and $\mathrm{N}$ groups $(P<0.00 \mathrm{I}$, Student's $t$-test), but not the $M$ group. When comparing the chronotype groups together, mean sleep duration was not significantly different between groups on weekdays, but was so on weekends ( $P=0.007$, analysis of variance).

Note: *Significant difference in sleep duration among the chronotype groups on weekends.

Abbreviations: $\mathrm{E}$, evening chronotype; $\mathrm{N}$, neither type; $\mathrm{M}$, morning chronotype.

\section{Employment}

Among the employed students, neither chronotype nor poor sleep quality was significantly related to work status. However, more of the employed students were evening types (24.8\%) and sleep-disturbed (66.2\%) compared to the unemployed students.

\section{Smoking}

There was a significant negative correlation between number of cigarettes smoked and MEQ ( $r=-0.094, P=0.050$ ), indicating an increased smoking rate with eveningness.
The number of cigarettes smoked correlated positively and significantly with PSQI global score $(r=0.162, P=0.018)$, implying that a greater amount of smoking is associated with poor sleep.

\section{Sleep habits and irregular bedtime schedules}

There was no significant relationship between PSQI and bedtime habits between weekdays and weekends. Out of those who had regular bedtime schedules across the week (less than 1-hour change in sleep timing between weekdays and weekends), $61.3 \%$ exhibited poor sleep compared to

Table 3 Variation of respondent sleep-wake habits and duration between weekdays and weekends with respect to circadian typology

\begin{tabular}{|c|c|c|c|c|c|c|}
\hline & \multicolumn{3}{|l|}{ Chronotypes } & \multirow[t]{3}{*}{$\boldsymbol{F}$} & \multirow[t]{3}{*}{$P$} & \multirow[t]{3}{*}{$\eta^{2} \rho$} \\
\hline & Evening & Neither & Morning & & & \\
\hline & Mean \pm SD & Mean \pm SD & Mean \pm SD & & & \\
\hline \multicolumn{7}{|l|}{ Wake-up time } \\
\hline Weekdays & $8: 33 \mathrm{am} \pm 90 \mathrm{~min}$ & $7: 44 \mathrm{am} \pm 82 \mathrm{~min}$ & $7: 04 \mathrm{am} \pm 62 \mathrm{~min}$ & 19.494 & $<0.00 I^{*}$ & 0.086 \\
\hline Weekends & $11: 38 \mathrm{am} \pm 97 \mathrm{~min}$ & $10: 04 \mathrm{am} \pm 101 \mathrm{~min}$ & $8: 16$ am $\pm 85 \mathrm{~min}$ & 61.084 & $<0.00 I^{*}$ & 0.224 \\
\hline \multicolumn{7}{|l|}{ Bedtime } \\
\hline Weekdays & $12: 47 \mathrm{am} \pm 80 \mathrm{~min}$ & $12: 01 \mathrm{am} \pm 74 \mathrm{~min}$ & $1 \mathrm{I}: 12 \mathrm{pm} \pm 87 \mathrm{~min}$ & 23.023 & $<0.00 I^{*}$ & 0.098 \\
\hline Weekends & $2: 37 \mathrm{am} \pm 82 \mathrm{~min}$ & $\mathrm{I}: 27 \mathrm{am} \pm 96 \mathrm{~min}$ & $12: 22 \mathrm{am} \pm 10 \mid \mathrm{min}$ & 33.258 & $<0.00 I^{*}$ & 0.137 \\
\hline \multicolumn{7}{|c|}{ Sleep duration (hours) } \\
\hline Weekdays & $7.78 \pm 1.48$ & $7.67 \pm 1.48$ & $7.86 \pm 1.12$ & 0.419 & 0.658 & 0.002 \\
\hline Weekends & $9.00 \pm 1.54$ & $8.62 \pm 1.79$ & $7.91 \pm 1.96$ & 4.957 & $0.007^{*}$ & 0.023 \\
\hline
\end{tabular}

Note: *Significant difference in sleep/wake timing among chronotype groups on weekdays and weekends.

Abbreviations: SD, standard deviation; min, minutes. 
$56.1 \%$ of poor sleepers among those with irregular bedtime schedules.

\section{Discussion}

To our knowledge, the present study is the first to assess sleep quality and habits among Lebanese university students in a culture-specific context. PSQI global scores indicated that poor sleep was common in this group of Lebanese university students, and a similar proportion of Lebanese students had PSQI scores indicating poor sleep quality, as in prior studies of North American ${ }^{22}$ and Chinese university students. ${ }^{36}$ However, studies in other cultures, such as South American, ${ }^{37,38}$ Asian, ${ }^{39,40}$ Ethiopian, ${ }^{41}$ and Palestinian, ${ }^{28}$ reported lower proportions of university students with poor sleep. While poor sleep appears to be quite common among university students, the reported variations between different studies may be influenced by different socioeconomic demands and cultural habits among the different population groups. ${ }^{42}$

Insufficient or poor-quality sleep has been demonstrated to be associated with adverse academic performance. ${ }^{38,43}$ Prior studies addressing the impact of sleep disturbance on students' performance and health risks have attributed poor sleep quality to alteration in one or more of the sleep domains. ${ }^{22,42,44,45}$ Such sleep dimensions include nocturnal sleep length and variability of sleep-wake schedules. In the present study, poor sleep quality was associated with reduced nighttime sleep duration, although the overall mean nocturnal sleep duration in our sample was nearly 8 hours. Only an eighth of the student respondents reported insufficient sleep according to our definition, with $1.6 \%$ sleeping fewer than 5 hours per night and $5.7 \%$ sleeping fewer than 6 hours per night. However, we observed an interaction between sleep duration on weekdays (when class-start times and academic demands had to be observed by students) versus weekends, and circadian typology. While sleep duration did not vary among the different chronotypes on weekdays, it was both prolonged and more variable between chronotypes on weekends, especially for evening-type subjects. This finding suggests a temporal misalignment between the biologically driven circadian rhythms and socially dictated behaviors on school nights that create a sleep debt in evening chronotypes, which they may attempt to compensate for on weekends when there are minimal academic or social constraints. Similar findings have been reported previously, whereby eveningtype university students showed partial sleep deprivation on weekdays secondary to "social jet lag". 14,46-48 While some studies have suggested that evening-type individuals have a greater sleep need, others found no chronotype influence on sleep need. ${ }^{49}$ Roepke and Duffy ${ }^{50}$ reported a very modest difference in self-reported sleep need between evening and morning types, and suggested a matching of sleep duration and sleep need across the week in morning types, whereas evening types accumulate a sleep deficit across the week and extend sleep to make up for it during the weekends. Our study therefore adds to the evidence that one important way in which circadian typology impacts sleep is through sleep duration.

Delays in sleep-wake timing between weekdays and weekends were notable among our respondents. Overall, our students delayed their bedtime by an average of 1.5 hours on weekends, much longer than the approximate half-hour weekend difference reported by Valencia-Flores et $\mathrm{al}^{51}$ in Mexican university students, and thus raising the potential influence of cultural habits on sleep regularities. However, one cannot exclude the potential contribution of biological determinants. Our study showed a significant correlation between chronotype and sleep-wake timing between weekdays and weekends. Although later weekend bedtimes were favored by all chronotypes in our study, conforming to a previous report by Crowley and Carskadon, ${ }^{52}$ our evening-type students exhibited significantly greater delays in bedtimes and wake times than morning or neither types between weekdays and weekends.

Prior studies have reported that regular and stable sleepwake schedules, generally observed among morning types, are associated with better subjective sleep quality, ${ }^{53}$ and that evening types are more prone to sleep disturbances. ${ }^{54-56} \mathrm{In}$ our study, chronotype significantly (negatively) correlated with PSQI global score, indicating a higher incidence of poor sleep with greater eveningness. Therefore, our findings provide further evidence of the impact of chronotype on sleep quality through affecting the variability of sleep-wake timing across the week.

Taken together, our findings suggest that chronotype interacts with the schedules of students to affect their sleep quality and duration. Within the context of Lebanese society where there is relatively little awareness of the importance of sleep, raising awareness about circadian typology and its potential impacts on daytime functioning could have important implications in academia and the workplace. Being informed about such issues may help students better design their class time and work schedule for optimal daytime performance.

We also investigated the impact of other behaviors and lifestyle habits, such as employment status and smoking, on sleep quality. Smoking, especially narghile, has increased greatly in Lebanon over the past decade. Accord- 
ing to one comparative study considering adolescent and adult behavioral habits in 17 different countries across the Americas, Europe, the Middle East, Africa, and Oceania, smoking was a major health-risk behavior in Lebanon. ${ }^{57}$ Our study revealed that more than half the university students we surveyed reported smoking cigarettes and/or narghile, and this behavior was significantly correlated with both poor sleep quality and eveningness. Mesquita et $\mathrm{al}^{58}$ reported that smoking was a major contributor to increased complaints of poor sleep quality among Brazilian university students. Additional studies suggesting the adverse impacts of tobacco use on sleep have been reported in other cultures, such as North America. ${ }^{59,60}$

Although the present study found no significant relationship between employment status and poor sleep quality and chronotype, we found that more of the working students were evening types, potentially adding to the list of social constraints that reduce their nocturnal sleep duration and contribute to their sleep debt.

In summary, the present study highlights the prevalence of poor sleep quality among university students in Lebanon. We found evidence that this poor sleep quality was associated with reduced sleep duration on weeknights and a shift in bedtimes on weekends, especially among evening-type students. It appears that both biological determinants (such as chronotype) and behavioral habits (such as smoking and employment status) interact to impact sleep in this population, affecting one or more of the sleep dimensions. This has important implications for the health and academic performance of Lebanese university students, as has been found for students in other countries. ${ }^{43,47,61,62}$ Raising awareness about the importance of sleep and the role of chronotype in obtaining adequate sleep may help Lebanese students make better choices in school and work schedules for optimal daytime and academic functioning.

\section{Study limitations}

Our results were based on retrospective self-reporting by students, which could have been subject to bias. The use of prospective measures, such as a sleep/work/health diary, and/or objective means of documenting sleep behaviors, such as actigraphy recording, may help elucidate these preliminary observations.

\section{Acknowledgments}

This study was supported by the Lebanese National Council for Scientific Research (CNRS-REF268). The authors thank Fr/Dr Bechara Khoury and Dr Jacqueline Doumit for their input in the early stages of drafting the proposal, and Ms Frida Torosyan for data entry.

\section{Disclosure}

The authors report no conflicts of interest in this work. JFD reports that she is a member of the Sleep Research Society Board of Directors. JFD was supported in part by the Brigham and Women's Hospital BRI Fund to Sustain Research Excellence.

\section{References}

1. Pilcher JJ, Ginter DR, Sadowsky B. Sleep quality versus sleep quantity: relationship between sleep and measures of health, well-being and sleepiness in college students. J Psychosom Res. 1997;42:583-596.

2. Van Dongen HP, Dinges DF. Investigating the interaction between the homeostatic and circadian processes of sleep-wake regulation for the prediction of waking neurobehavioral performance. J Sleep Res. 2003; 12:181-187.

3. Borbely AA. A two-process model of sleep regulation. Human Neurobiol. 1982;1:195-204.

4. Grandner MA, Sands-Lincoln MR, Pak VM, Garland SN. Sleep duration, cardiovascular disease, and proinflammatory biomarkers. Nat Sci Sleep. 2013;5:93-107.

5. Spiegel K, Leproult R, Van Auter E. Impact of sleep debt on metabolic and endocrine function. Lancet. 1999;354:1435-1439.

6. Wingard DL, Berkman LF. Mortality risk associated with sleeping patterns among adults. Sleep. 1983;6:102-107.

7. Millman RP. Excessive sleepiness in adolescents and young adults: causes, consequences, and treatment strategies. Pediatrics. 2005;115 1774-1786.

8. Carskadon MA. Patterns of sleep and sleepiness in adolescents Pediatrician. 1990;17:5-12.

9. Carskadon MA, Vieira C, Acebo C. Association between puberty and delayed phase preference. Sleep. 1993;16:258-262.

10. Valdez P, Ramírez C, García A. Delaying and extending sleep during weekends: sleep recovery or circadian effect? Chronobiol Int. 1996;13 191-198.

11. Kerkhof GA, Van Dongen HP. Morning-type and evening-type individuals differ in the phase position of their endogenous circadian oscillator. Neurosci Lett. 1996;218:153-156.

12. Adan A, Archer SN, Hidalgo MP, Milia LD, Natale V, Randler C. Circadian typology: a comprehensive review. Chronobiol Int. 2012;29: 1153-1175.

13. Borchers C, Randler C. Sleep-wake cycle of adolescents in Cote d'Ivoire: influence of age, gender, religion and occupation. Chronobiol Int. 2012;29:1366-1375.

14. Wittmann M, Dinich J, Merrow M, Roenneberg T. Social jetlag: misalignment of biological and social time. Chronobiol Int. 2006;23: 497-509.

15. Smith MR, Eastman CI. Shift work: health, performance and safety problems, traditional countermeasures, and innovative management strategies to reduce circadian misalignment. Nat Sci Sleep. 2012;4:111-132.

16. Korczak AL, Martynhak BJ, Pedrazzoli M, Brito AF, Louzada FM. Influence of chronotype and social zeitgebers on sleep/wake patterns. Braz J Med Biol Res. 2008;41:914-919.

17. Patel NP, Grandner MA, Xie D, Branas CC, Goonertne N. "Sleep disparity" in the population: poor sleep quality is strongly associated with poverty and ethnicity. BMC Public Health. 2010;10:475-485.

18. Schlarb AA, Kulessa D, Gulewitsch MD. Sleep characteristics, sleep problems, and associations of self-efficacy among German university students. Nat Sci Sleep. 2012;4:1-7.

19. Hicks RA, Fernandez C, Pelligrini RJ. Striking changes in the sleep satisfaction of university students over the last two decades. Percept Mot Skills. 2001;93:660. 
20. Horne JA. Is there a sleep debt? Sleep. 2004;27:1047-1049.

21. Hosek CA, Phelps BJ, Jensen J. Average sleep times among undergraduate college students. Psi Chi J Undergrad Res. 2004;9:57-61.

22. Lund HG, Reider BD, Whiting AB, Prichard JR. Sleep patterns and predictors of disturbed sleep in a large population of college students. J Adolesc Health. 2010;46:124-132.

23. Wiebe ST, Cassoff J, Gruber R. Sleep patterns and the risk of unipolar depression: a review. Nat Sci Sleep. 2012;4:63-71.

24. Curcio G, Ferrara M, De Gennaro L. Sleep loss, learning capacity and academic performance. Sleep Med Rev. 2006;10:323-337.

25. Dewald JF, Meijer AM, Oort FJ, Kerkhof GA, Bogels SM. The influence of sleep quality, sleep duration and sleepiness on school performance in children and adolescents: a meta-analytic review. Sleep Med Rev. 2010;14:179-189.

26. Durmer JS, Dinges DF. Neurocognitive consequences of sleep deprivation. Semin Neurol. 2005;25:117-129.

27. Kelly WE, Kelly KE, Clanton RC. The relationship between sleep length and grade point average among college students. Coll Stud J. 2001;35:84-86.

28. Sweileh WM, Ali IA, Sawalha AF, Abu-Taha AS, Zyoud SH, Al-Jabi SW. Sleep habits and sleep problems among Palestinian students. Child Adolesc Psychiat Mental Health. 2011;5:25-33.

29. BaHammam A. Sleep pattern, daytime sleepiness, and eating habits during the month of Ramadan. Sleep Hypn. 2003;5:165-172.

30. Jean-Louis G, Magai CM, Cohen CI, et al. Ethnic differences in selfreported sleep problems in older adults. Sleep. 2001;24:926-933.

31. Jean-Louis G, Zizi F, Casimir G, Compas J. Sleep in America: is race or culture an important factor? In: Léger D, Pandi-Perumal SR, editors. Sleep Disorders: Their Impact on Public Health. Boca Raton (FL): CRC; 2007.

32. Yahia N, Achkar A, Abdallah A, Rizk S. Eating habits and obesity among Lebanese university students. Nutr J. 2008;7:32-37.

33. Shochat T. Impact of lifestyle and technology developments on sleep. Nat Sci Sleep. 2012;4:19-31.

34. Buysse DJ, Reynolds CF 3rd, Monk TH, Berman SR, Kupfer DJ. The Pittsburgh Sleep Quality Index: a new instrument for psychiatric practice and research. Psychiatry Res. 1989;28:193-213.

35. Horne JA, Ostberg O. A self-assessment questionnaire to determine morningness-eveningness in human circadian rhythms. Int $J$ Chronobiol. 1976;4:97-110.

36. Suen LK, Ellis Hon LK, Tam WW. Association between sleep behavior and sleep-related factors among university students in Hong Kong. Chronobiology Int. 2008;25:760-775.

37. Lima AM, Varela GC, Silveira HA, Parente RD, Araujo JF. Evening chronotypes experience poor sleep quality when taking classes with early starting times. Sleep Sci. 2010;3:45-48.

38. Medeiros AL, Mendes DB, Lima PF, Araujo JF. The relationship between sleep-wake cycle and academic performance in medical students. Biol Rhythm Res. 2001;32:263-270.

39. Cheng SH, Shih CC, Lee IH, et al. A study on the sleep quality of incoming university students. Psychiatry Res. 2012;197:270-274.

40. Kang JH, Chen CS. Effects of an irregular bedtime schedule on sleep quality, daytime sleepiness, and fatigue among university students in Taiwan. BMC Public Health. 2009;9:248-254.

41. Lemma S, Patel SV, Tarekegn YA, et al. The epidemiology of sleep quality, sleep patterns, consumption of caffeinated beverages, and khat use among Ethiopian college students. Sleep Disord. 2012;2012:1-11.

Nature and Science of Sleep

\section{Publish your work in this journal}

Nature and Science of Sleep is an international, peer-reviewed, open access journal covering all aspects of sleep science and sleep medicine, including the neurophysiology and functions of sleep, the genetics of sleep, sleep and society, biological rhythms, dreaming, sleep disorders and therapy, and strategies to optimize healthy sleep. The journal welcomes
42. Janson C, Gislason T, De Backer W, et al. Prevalence of sleep disturbances among young adults in three European countries. Sleep. 1995; 18:589-597.

43. Gomes AA, Tavares J, de Azevedo MH. Sleep and academic performance in undergraduates: a multi-measure, multi-predictor approach. Chronobiol Int. 2011;28:786-801.

44. Lima PF, Medeiros AL, Rolim SA, Dias SA, Almondes KM, Araujo JF. Changes in sleep habits of medical students according to class starting time: a longitudinal study. Sleep Sci. 2009;2:92-95

45. Suen KP, Tam WS, Hon KL. Association of sleep hygiene-related factors and sleep quality among university students in Hong Kong. Hong Kong Med J. 2010;16:180-185.

46. Adan A, Natale V, Caci H, Prat G. Relationship between circadian typology and functional and dysfunctional impulsivity. Chronobiol Int. 2010;27:620-639.

47. Besoluk S, Onder I, Deveci I. Morningness-eveningness preferences and academic achievement of university students. Chronobiol Int. 2011;28:118-125.

48. Taylor DJ, Clay KC, Bramoweth AD, Sethi K, Roane BM. Circadian phase preference in college students: relationship with psychological functional academics. Chronobiol Int. 2011;28:541-547.

49. Taillard J, Philip P, Bioulac B. Morningness/eveningness and the need for sleep. J Sleep Res. 1999;8:291-295.

50. Roepke SE, Duffy JF. Differential impact of chronotype on weekday and weekend sleep timing and duration. Nat Sci Sleep. 2010;2: 213-220.

51. Valencia-Flores M, Castaño VA, Campos RM, et al. The siesta culture concept is not supported by the sleep habits of urban Mexican students. J Sleep Res. 1998;7:21-29.

52. Crowley SJ, Carskadon MA. Modifications to weekend recovery sleep delay phase in older adolescents. Chronobiol Int. 2010;27:1469-1492.

53. Ikeda H, Hayashi M. Longitudinal study of self-awakening and sleep/ wake habits in adolescents. Nat Sci Sleep. 2012;4:103-109.

54. Monk TH, Reynolds, CF, Buysse DJ, DeGrazia JM, Kupfer DJ. The relationship between lifestyle regularity and subjective sleep quality. Chronobiol Int. 2003;20:97-107.

55. Monk TH, Buysse DJ, Potts JM, DeGrazia JM, Kupfer DJ. Morningnesseveningness and lifestyle regularity. Chronobiol Int. 2004;21:435-443.

56. Soehner AM, Kennedy KS, Monk TH. Circadian preference and sleep-wake regularity: associations with self-reported parameters in daytime-working adults. Chronobiol Int. 2011;28:802-809.

57. Degenhardt L, Chiu WT, Sampson N, et al. Toward a global view of alcohol, tobacco, cannabis, and cocaine use: findings from the WHO World Mental Health Surveys. PLos Med. 2008;5:e141.

58. Mesquita G, Ferreira S, Rossini S, Soares EA, Reimao R. Effects of tobacco and alcohol consumption on sleep quality of university students. Neurobiologia. 2011;74:19-27.

59. Phillips BA, Danner FJ. Cigarette smoking and sleep disturbance. Arch Intern Med. 1995;155:734-737.

60. Zhang L, Samet J, Caffo B, Punjabi NM. Cigarette smoking and nocturnal sleep architecture. Am J Epidemiol. 2006;164:529-537.

61. Buysse DJ, Angst A, Ajdacic V, Eich D, Rossler W. Prevalence, course, and comorbidity of insomnia and depression in young adults. Sleep. 2008;31:473-480.

62. Knutson KL, Van Cauter E, Rathouz PJ, et al. Association between sleep and blood pressure in midlife: the CARDIA sleep study. Arch Intern Med. 2009;169:1055-1061.

\section{Dovepress}

original research, clinical \& epidemiological studies, reviews \& evaluations, case reports and extended reports. The manuscript management system is completely online and includes a very quick and fair peerreview system, which is all easy to use. Visit http://www.dovepress.com/ testimonials.php to read real quotes from published authors. 